Military Technical College

Kobry El-Kobba

Cairo, Egypt

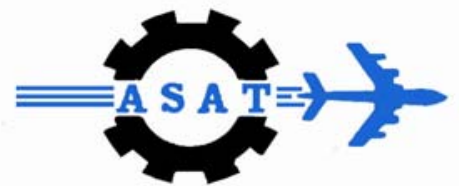

12-th International Conference

on

Aerospace Sciences \&

Aviation Technology

\title{
INVERSE SIMULATION WITH FEED BACK TECHNIQUE FOR AIRCRAFT MOTION ALONG PRESCRIBED TRAJECTORIES
}

\author{
Elbahnasy ${ }^{*}$ I.S
}

\begin{abstract}
This paper is a trial to establish global results for inverse simulation technique as applied to aircraft. This work builds on a previous result for rigid aircraft inverse simulation and adds a new level of complexity, that the aircraft is subjected to external sudden disturbances. The external sudden disturbance cannot be ignored even in the most basic flight conditions, thus the feed back will overcome this disturbances effects on aircraft dynamics and interprets that into modification of the control inputs. The technique was applied to some interesting two dimensional and three dimensional maneuvers.
\end{abstract}

\section{KEYWORDS}

Aircraft, Inverse Simulation, Dynamics and Control

\section{NOMENCLATURE}

\begin{tabular}{|c|c|c|c|}
\hline b & $=$ Wing span & $u, v, w$ & $\begin{array}{l}=\text { Forward, side, and downward } \\
\text { velocities }\end{array}$ \\
\hline $\mathrm{C}$ & $=$ Mean aerodynamic chord & $X, Y, Z$ & $=$ Aircraft coordinate w.r.t inertial axes \\
\hline$C_{L}, C_{D}, C_{Y}$ & $\begin{array}{l}=\text { Lift }, \text { drag and side force } \\
\text { coefficients }\end{array}$ & $\alpha$ & $=$ Angle of attack \\
\hline $\mathrm{C}_{1}, \mathrm{C}_{\mathrm{m}}, \mathrm{C}_{\mathrm{n}}$ & $\begin{array}{l}=\text { Roll, pitch and yaw moment } \\
\text { coefficients }\end{array}$ & $\beta$ & $=$ Sideslip slip angle \\
\hline F & $=$ Applied force & $\delta a, \delta e, \delta r$ & $\begin{array}{l}=\begin{array}{l}\text { Aileron, elevator and rudder } \\
\text { deflection }\end{array}\end{array}$ \\
\hline $\begin{array}{l}G \\
H_{B}\end{array}$ & $\begin{array}{l}=\text { Gravity acceleration } \\
=\text { Angular momentum }\end{array}$ & $\begin{array}{l}\psi, \Theta, \varphi \\
\omega\end{array}$ & $\begin{array}{l}=\text { Attitude angles (yaw, } \text { pitch and roll) } \\
=\text { Angular velocity vector of body axes }\end{array}$ \\
\hline I & $=$ Inertia matrix & \multicolumn{2}{|c|}{ Superscript } \\
\hline $\mathrm{K} 1, \mathrm{k} 2, \mathrm{k} 3, \mathrm{k} 4$ & $=$ Control gains & . & $=$ Time derivative \\
\hline $\begin{array}{l}\mathrm{M} \\
\mathrm{Q} \\
\mathrm{p}, \mathrm{q}, \mathrm{r}\end{array}$ & $\begin{aligned}= & \text { Applied moment } \\
= & \text { Dynamic pressure } \\
= & \text { Roll, pitch and yaw angular } \\
& \text { velocities }\end{aligned}$ & $\begin{array}{l}\text { T } \\
-1 \\
\text { Subscript }\end{array}$ & $\begin{array}{l}=\text { Transpose of a matrix } \\
=\text { Inverse of a matrix }\end{array}$ \\
\hline $\begin{array}{l}S \\
T\end{array}$ & $\begin{array}{l}=\text { Wing area } \\
=\text { Thrust force }\end{array}$ & $\begin{array}{ll}0 \\
T\end{array}$ & $\begin{array}{l}=\text { Initial value } \\
=\text { Target }\end{array}$ \\
\hline $\mathrm{V}_{\mathrm{B}}$ & $=$ Velocity vector & & \\
\hline
\end{tabular}

\footnotetext{
${ }^{*}$ Flight Test Engineer, Aerospace Research Center, Cairo, Egypt Flight Dynamics Engineer, Aerospace Research Center, Cairo Egypt Graduate Student, Aerospace Department, Cairo University, Giza, Egypt
} 


\section{INTRODUCTION}

Inverse simulation is a technique used to determine the control inputs that enable a dynamic system, such as aircraft to produce desired output. Recently, inverse simulation technique has gained considerable popularity in application to highly maneuverable aircraft [1].

In virtue of this interest in some aspects of the inverse problem of aircraft control, a general and simple formulation of the problem was given by Abdel Rahman and $\mathrm{Al}$ Bahi [2] and was applied for the case of circular vertical loop and spatial maneuvers. Fisher [3] implements the control methodology of the aircraft attitude as an outer loop controller to an aircraft under nonlinear dynamic inversion control. Instead a control algorithm for both attitude and position was proposed here. Karlsson [4] cancels the non-linear dynamics and then the system can be controlled as a linear system while, for the present study nonlinear dynamics was considered.

The present study represents the technique of inverse simulation, based on the calculation of required forces and moments to perform certain maneuver using feed back control "error minimizing technique [1-2]" as shown in Fig. (1), which makes the problem similar to control allocation problem.

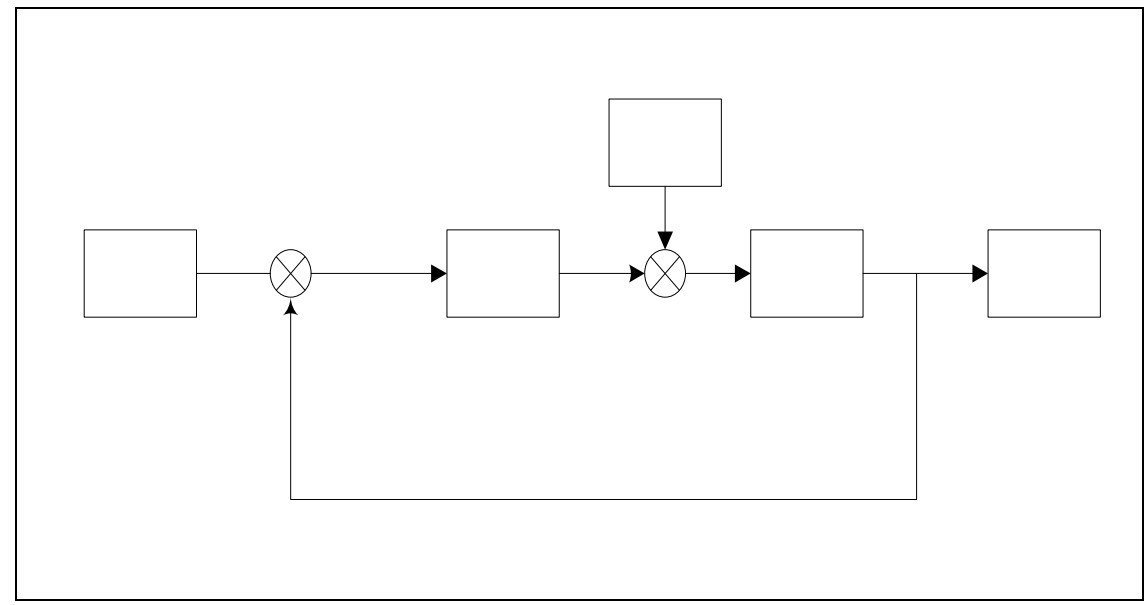

Fig.1. Feed Back Control Technique

This study is different from previous studies in that, it includes the feed back control algorithm for both attitude and position which gives the techniques the ability to present the required control laws in case of sudden or even continuous disturbances and maintains the required trajectory.

The paper is organized as follows. The first section shows the general formulation of the technique and describes the equations of motion for the aircraft followed by a definition of control algorithm which calculates the required control forces and moments in section two. Section three presents the mathematical model of the external forces and moments. Section four shows the calculation procedure with numerical application results for both two dimensional and three dimensional maneuvers, followed by concluding remarks. 


\section{GENERAL FORMULATION}

Rigid aircraft motion is generally represented by the six components coordinate vector in addition to the six components quasi velocity vector [2].

Elements of both vectors can be gathered in the vector $\zeta$

$$
\zeta=[\mathbf{V}, \omega, \mathbf{x}, \theta]
$$

Where $V=$ velocity, $\omega=$ angular velocity

$\mathrm{x}=$ position, $\theta$ =attitude angles

Similarly the target maneuver is given in the following form

$$
\zeta_{\mathrm{T}}=\left[\mathbf{V}_{\mathrm{T}}, \omega_{\mathrm{T}}, \mathbf{x}_{\mathrm{T}}, \theta_{\mathrm{T}}\right]
$$

The subscript T means target maneuver

The total required forces and moments to perform the required maneuver is calculated based on the difference between the given maneuver parameters and the six degree of freedom model output [5]

Which can be presented as :

$$
\mathbf{F}_{\mathbf{C}}=\mathbf{K}\left[\zeta_{\mathbf{T}}-\zeta\right]
$$

Where $\mathrm{K}=$ gain matrix which is chosen according to the required dynamic response.

From which we see that the value of $F_{C}$ is increased when the difference between the required maneuver parameter and real aircraft state increases.

The forces acting on the aircraft are: gravity, aerodynamic forces and thrust [6]. So the total forces and moments necessary to perform the required maneuver is a combination of the gravity, aerodynamic and thrust forces and moments

Since the gravity forces is known and can be calculated in the body axes so later we will call the combination of aerodynamic forces and thrust as $F_{A C}$

Since $F_{A C}=$ aerodynamic force + thrust force

And the aerodynamic forces and moments are functions of the flow angles and the control surface deflections

$$
\mathbf{F}_{\mathrm{AC}}=\mathbf{f}(\boldsymbol{\alpha}, \boldsymbol{\beta}, \boldsymbol{\delta} \mathbf{e}, \boldsymbol{\delta} \mathbf{a}, \boldsymbol{\delta} \mathbf{r}, \mathbf{T})
$$

As we have already calculated $F_{C}$ and gravity forces are known so we have six unknown for six equations which can be solved to get the matrix column $\delta=$ $[\alpha, \beta, \delta \mathrm{e}, \delta \mathrm{a}, \delta \mathrm{r}, \mathrm{T}]$.

\section{AIRCRAFT EQUATIONS OF MOTION}

The nonlinear aircraft six degree of freedom dynamic model depend on representing the dynamics of the system with usage of two sets of axes as shown in Fig. (2). -inertial axes system which is fixed to earth surface and we neglect the earth rotation about itself

-body fixed axes which is fixed to aircraft body under all conditions 


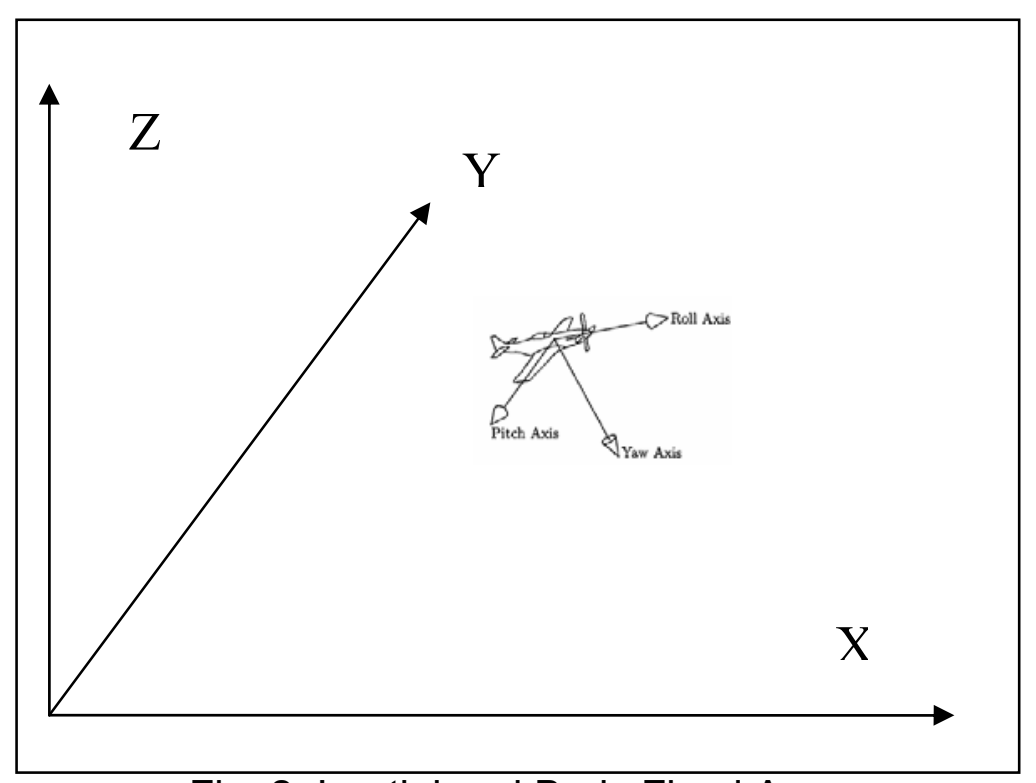

Fig. 2. Inertial and Body Fixed Axes

The equation of motion for the aircraft can be written down in accordance with the Newtonian laws of motion [6]

Where $\mathrm{H}_{\mathrm{B}}=\mathrm{I} \omega$

$$
\begin{aligned}
& F=m\left(\dot{V}_{B}+\omega \times V_{B}\right) \\
& M=\dot{H}_{B}+\omega \times H_{B}
\end{aligned}
$$

Rearrange the above two equations

$$
\begin{aligned}
& \dot{V}_{B}=\frac{F}{m}-\omega \times V_{B} \\
& \dot{\omega}=[I]^{-1}(M-\omega \times I \omega)
\end{aligned}
$$

This is equivalent to six equations in six unknowns. Equation (7) can be solved independent of equation (6) but equation (6) can't be solved independent of equation (7), which physically means that the aircraft attitude "or any rigid body" can be determined independent of aircraft position but aircraft position can't be determined without determination of aircraft attitude.

Integrating the above equation using $4^{\text {th }}$ order Runge Kutta method for given initial Condition of $\omega \& V_{B}$ so we get

$\omega=\left[\begin{array}{l}p \\ q \\ r\end{array}\right], \mathrm{V}_{\mathbf{B}}=\left[\begin{array}{l}u \\ v \\ w\end{array}\right]$

To get the position \& attitude of the aircraft relative to inertial axes we use kinematics relations which transfer the above values of velocities and angular velocities from body axes into inertial axes. 
The Euler angles technique is used for transformation between the inertial and body axes, The kinematics relation is as follows [5]:

$$
\begin{aligned}
& \dot{\psi}=(q \sin \phi+r \cos \phi) / \cos \theta \\
& \dot{\theta}=q \cos \phi-r \sin \phi \\
& \dot{\phi}=(q \sin \phi+r \cos \phi) \tan \theta+p \\
& \dot{X}=u \cos \psi \cos \theta+v(-\sin \psi \cos \phi+\cos \psi \sin \phi \sin \theta) \\
& \quad+w(-\sin \psi \sin \phi+\cos \psi \cos \phi \sin \theta) \\
& \dot{Y}=u \sin \psi \cos \theta+v(-\cos \psi \cos \phi+\sin \psi \sin \phi \sin \theta) \\
& \quad+w(-\cos \psi \sin \phi+\cos \psi \cos \phi \sin \theta) \\
& \dot{Z}=-u \sin \psi \cos \theta+v \sin \phi \cos \theta+w \cos \phi \cos \theta
\end{aligned}
$$

By solving "integrating" the above six equation using $4^{\text {th }}$ order Runge Kutta- 4 method together with equations(1) and (2) at given initial Conditions of $\psi, \Theta, \varphi, \mathrm{x}, \mathrm{y}, \mathrm{z}$ so the position and attitude of the aircraft is completely defined.

\section{CALCULATIONS OF NECESSARY FORCES AND MOMENTS TO PERFORM THE TARGET MANEUVER}

the calculations of necessary forces and moments to perform certain maneuver is simply depends on the criteria that we need only force to change the position and only moment to change the attitude. By application of PD control "proportional differential controller" [5] which is not only reduce the error in the required state but also in its derivative the elements of control forces and moments vector will be as follows :

$$
\begin{aligned}
& F c x=k 1\left(x_{T}-x\right)+k 2\left(u_{T}-u\right) \\
& \mathrm{Fcy}=\mathrm{k} 1\left(\mathrm{y}_{\mathrm{T}}-\mathrm{y}\right)+\mathbf{k} 2\left(\mathrm{v}_{\mathrm{T}}-\mathrm{v}\right) \\
& \mathrm{Fcz}=\mathrm{k} 1\left(\mathrm{z}_{\mathrm{T}}-\mathrm{z}\right)+\mathrm{k} 2\left(\mathrm{w}_{\mathrm{T}}-\mathrm{w}\right) \\
& \operatorname{Mcx}=k 3\left(\varphi_{T^{-}} \varphi\right)+k 4\left(p_{T^{-}} p\right) \\
& \mathrm{Mcy}=\mathrm{k3}\left(\Theta_{\mathrm{T}^{-}} \boldsymbol{\theta}\right)+\mathrm{k} 4\left(\mathrm{q}_{\mathrm{T}^{-}}-\mathrm{q}\right) \\
& M c z=k 3\left(\psi_{T^{-}} \psi\right)+k 4\left(r_{T^{-}}\right)
\end{aligned}
$$

So

$$
\mathrm{F}_{\mathrm{c}}=[\mathrm{Fcx}, \mathrm{Fcy}, \mathrm{Fcz}, \mathrm{Mcx}, \mathrm{Mcy}, \mathrm{Mcz}]
$$

which calculated or designed based on required transient response characteristics such as settling time, damping ratio, percentage overshoot and natural frequency As mentioned before subscript $T$ means the target maneuver, and $x, y, z$ subscripts refers to the direction of the force or the moment.

\section{MATHEMATICAL MODEL OF EXTERNAL FORCES AND MOMENTS}

The external forces and moments acting on the aircraft during flight is divided into three categories first is the aerodynamic forces and moments; second is the gravity 
forces and finally the thrust force "in some cases the thrust also causes some moments such as if the thrust line is deviated from the center of gravity".

\section{i-Aerodynamic Forces and Moments}

The aerodynamic forces and moments are assumed to be linear and they are represented in the stability body axes system. Stability axes system is defined as follows:

the $\mathrm{X}$ axis is in the direction of flight "the direction of velocity vector" so it differs with the variation of angle of attack $\alpha$ or sideslip angle $\beta$, while $Z$ axis is in downward direction perpendicular to $X$ axis in the aircraft plane of symmetry, and finally $Y$ axis is normal to XZ plane following the right hand rule "in the right wing direction" [5].

Aerodynamic forces are divided into 3 forces and 3 moments they are (drag "D", lift "L", side force "Y", rolling moment " $\ell$ ", pitching moment "m" and yawing moment "n") respectively [7].

$$
\begin{aligned}
& \mathrm{F}_{\mathrm{Xs}}=-\mathrm{D}=-\mathrm{Qs}\left(\mathrm{C}_{\mathrm{D} 0}+\mathrm{C}_{\mathrm{D} \alpha} \alpha+\mathrm{C}_{\mathrm{D} \delta \mathrm{e}} \delta \mathrm{e}+\mathrm{C}_{\mathrm{Dq}} \frac{q c}{2 V}\right) \\
& \mathrm{F}_{\mathrm{Ys}}=\mathrm{Y}=\mathrm{Qs}\left(\mathrm{C}_{\mathrm{y} \beta} \beta+\mathrm{C}_{\mathrm{y} \delta \mathrm{a}} \delta \mathrm{a}+\mathrm{C}_{\mathrm{y} \delta \mathrm{r}} \delta \mathrm{r}^{+} C_{y_{p}} \frac{p b}{2 V}+C_{y_{r}} \frac{r b}{2 V}\right) \\
& \mathrm{F}_{\mathrm{Zs}}=-\mathrm{L}=-\mathrm{Qs}\left(\mathrm{C}_{\mathrm{L} \cdot}+\mathrm{C}_{\mathrm{L} \delta \mathrm{e}} \delta \mathrm{e}+\mathrm{C}_{\mathrm{L} \alpha} \alpha+C_{L q} \frac{q c}{2 V}\right) \\
& \mathrm{M}_{\mathrm{Xs}}=\ell=\mathrm{Qsb}\left(C_{\ell_{\delta}} \delta a+C_{\beta} \beta+C \ell_{\delta} \delta r+C_{l r} \frac{r b}{2 V}+C_{l p} \frac{p b}{2 V}\right) \\
& \mathrm{M}_{\mathrm{Ys}}=\mathrm{m}=\mathrm{Qsc}\left(\mathrm{C}_{\mathrm{m} 0}+\mathrm{C}_{\mathrm{m} \alpha} \alpha+\mathrm{C}_{\mathrm{m} \delta \mathrm{e}} \delta \mathrm{e}^{+} C_{m q} \frac{q c}{2 V}\right) \\
& \mathrm{M}_{\mathrm{Zs}}=\mathrm{n}=\mathrm{Qsb}\left(\mathrm{C}_{\mathrm{n} \delta \mathrm{a}} \delta \mathrm{a}+\mathrm{C}_{\mathrm{n} \delta \mathrm{r}} \delta \mathrm{r}+\mathrm{C}_{\mathrm{n} \beta} \beta+{ }_{C_{r r}} \frac{r b}{2 V}+C_{n p} \frac{n b}{2 V}\right)
\end{aligned}
$$

\section{ii-Gravity Forces}

The gravity forces in the three directions $X, Y, Z$ respectively are as follows [6]:

$$
\text { Gravity force }=\left[\begin{array}{c}
-m g \sin \theta \\
m g \cos \theta \sin \phi \\
m g \cos \theta \cos \phi
\end{array}\right]
$$

\section{iii- Thrust Forces and Moments}

Finally the thrust force and moment are varying depending on location of the thrust line of each aircraft, but in most cases it is aligned to the body fixed $X$ axis and bathing through the center of gravity or very near to it, so the moment produced is very small and can be neglected.

\section{CALCULATION PROCEDURE}

First of all we should split the motion into longitudinal and lateral motion The longitudinal motion include the normal force, axial force and pitching moment while the lateral motion include the side force, rolling moment and yawing moment The forces and moments in the longitudinal motion 
can be presented in matrix form as follows:

$$
F_{\text {AC Long }}=Q^{*} S\left(A_{\text {flong }} \delta_{\text {Long }}+A_{\text {flong } 0}\right)
$$

Where

$$
\begin{aligned}
A_{\text {flong }}= & {\left[\begin{array}{ccc}
C_{D \alpha} & C_{D \delta e} & 1 /(Q * S) \\
C^{*} C_{m \alpha} & C^{*} C_{m \delta e} & 0 \\
C_{L \alpha} & C_{L \delta e} & 0
\end{array}\right], \delta_{\text {Long }}=\left[\begin{array}{c}
\alpha \\
\delta e \\
T
\end{array}\right] } \\
A_{\text {flong } 0} & =\left[\begin{array}{c}
C_{D 0}+C_{D q} \frac{\mathrm{qc}}{2 V} \\
C^{*} C_{m 0}+C^{*} C_{m q} \frac{\mathrm{qc}}{2 V} \\
C_{L 0}+C_{L q} \frac{\mathrm{qc}}{2 V}
\end{array}\right]
\end{aligned}
$$

The forces and moments in the lateral motion can be presented in matrix form as follows:

$$
F_{\text {AC Lateral }}=Q * S\left(A_{\text {fLateral }} \delta_{\text {Lateral }}+A_{\text {fLateral }}\right)
$$

where

$$
\begin{aligned}
A_{f L a t e r a l}= & {\left[\begin{array}{ccc}
b^{*} C_{l \beta} & b^{*} C_{l \delta a} & b^{*} C_{l \delta r} \\
C_{y \beta} & C_{y \delta a} & C_{y \delta r} \\
b^{*} C_{n \beta} & b^{*} C_{n \delta a} & b^{*} C_{n \delta r}
\end{array}\right], \delta_{\text {Lateral }}=\left[\begin{array}{c}
\beta \\
\delta a \\
\delta r
\end{array}\right] } \\
A_{f \text { fateral } 0}= & {\left[\begin{array}{c}
b^{*} C_{l r} \frac{r b}{2 V}+b^{*} C_{l p} \frac{p b}{2 V} \\
C_{y r} \frac{r b}{2 V}+C_{y p} \frac{p b}{2 V} \\
b^{*} C_{n r} \frac{r b}{2 V}+b^{*} C_{n p} \frac{p b}{2 V}
\end{array}\right] }
\end{aligned}
$$

Note that $p, q, r$ "body angular velocities" have been already determined from 6 degrees of freedom model.

The above formulas of aerodynamic forces are calculated in the stability axes so they should be transformed into the body axes. The next step after determination of aerodynamic forces and moments in the stability axes is to transform them into the body fixed axes in terms of sideslip angle and angle of attack.

The transformation matrix from stability axes to body fixed axes is as follows:

$$
T_{S}^{B}=\left[\begin{array}{ccc}
\cos \alpha \cos \beta & -\cos \alpha \sin \beta & -\sin \alpha \\
\sin \alpha \sin \phi \cos \beta+\sin \beta \cos \phi & -\sin \beta \sin \alpha \sin \phi+\cos \beta \cos \phi & \sin \phi \cos \alpha \\
\sin \alpha \cos \phi \cos \beta-\sin \beta \sin \phi & -\sin \beta \sin \alpha \cos \phi-\cos \beta \sin \phi & \cos \phi \cos \alpha
\end{array}\right]
$$


Now from above the system of equations will be:

$F_{\text {AC Long }}=Q * S * T_{S}^{B}\left(A_{\text {flong }} \delta_{\text {Long }}+A_{\text {flong } 0}\right)$

solving for $\delta_{\text {Long }}$ and rearrange

$$
\delta_{\text {Long }}=A_{\text {flong }}{ }^{-1}\left(\left[T_{S}^{B}\right]^{-1} \frac{F_{\text {AC Long }}}{Q * S}-A_{\text {flong } 0}\right) .
$$

And for lateral motion will be as follows

$$
\begin{aligned}
& F_{\text {AC Lateral }}=Q * S\left(A_{\text {fLateral }} \delta_{\text {Lateral }}+A_{\text {fLateral } 0}\right) \\
& \text { solving for } \delta_{\text {Lateral }} \text { and rearrange } \\
& \delta_{\text {Lateral }}=A_{\text {fLateral }}{ }^{-1}\left(\left(T_{S}^{B}\right)^{-1} * \frac{F_{A C \text { Lateral }}}{Q * S}-A_{\text {fLateral }_{0}}\right)
\end{aligned}
$$

Now we have $T_{S}^{B}$ which is calculated as a function of $\alpha$ and $\beta$ while $\alpha$ and $\beta$ are outputs from $\delta_{\text {Long }}$ and $\delta_{\text {Lateral }}$, to overcome This problem iteration technique has been developed which based on initially calculating $\alpha$ and $\beta$ [6] and solve equations (15) and (16) for new values of $\alpha$ and $\beta$ until the values of $\alpha$ and $\beta$ are fixed.

$u=U_{0} \cos \alpha \cos \beta$
$v=U_{0}(\sin \alpha \sin \phi \cos \beta+\sin \beta \cos \phi)$
$w=U_{0}(\sin \alpha \cos \phi \cos \beta-\sin \beta \sin \phi)$

where $U_{0}=\sqrt{u^{2}+v^{2}+w^{2}}$

Rearrange and solve for $\alpha$ and $\beta$

$$
\begin{aligned}
& \beta=\sin ^{-1}\left(\frac{v \cos \phi-w \sin \phi}{U_{0}}\right) \\
& \alpha=\cos ^{-1}\left(\frac{u}{U_{0}(\cos \beta)}\right)
\end{aligned}
$$

Note that the formulas show that both angle of attack and sideslip angle depend on the velocity values in the body fixed axes and rolling angle which were determined from the solution of aircraft six degree of freedom model.

\section{NUMERICAL APPLICATIONS}

In order to demonstrate the applicability of the inverse simulation technique with application of feed back control two cases of maneuvers were considered, first case 
is simple longitudinal $(x, z)$ plane motion while the second case is three dimensional maneuver which include both longitudinal and lateral motion.

Advanced trainer aircraft similar to German (alpha jet) was considered in the application [8].

Two Dimensional Application (Straight Line Vertical Loop Straight Line With Constant Speed).

The maneuver was performed with constant speed V=200 m/s. The maneuver shape and trajectory with time are shown in Figs $(3,4,5)$.

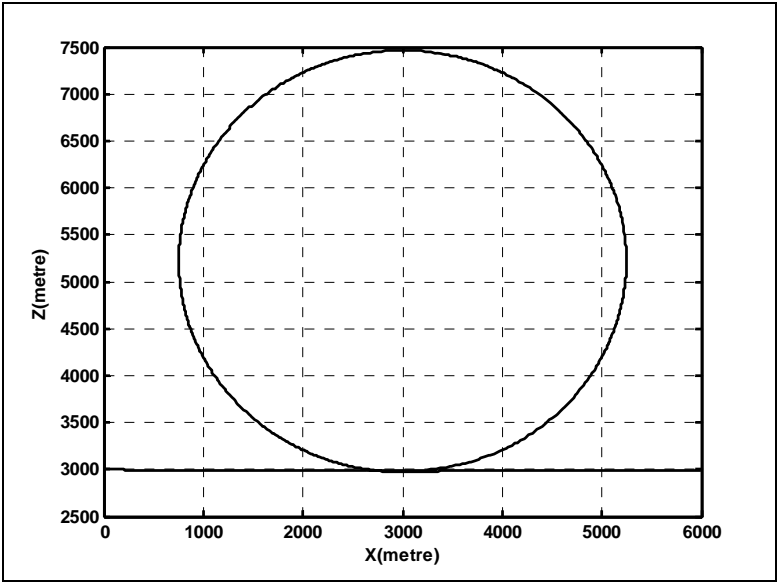

Fig.3. Straight-Vertical Loop-Straight Maneuver

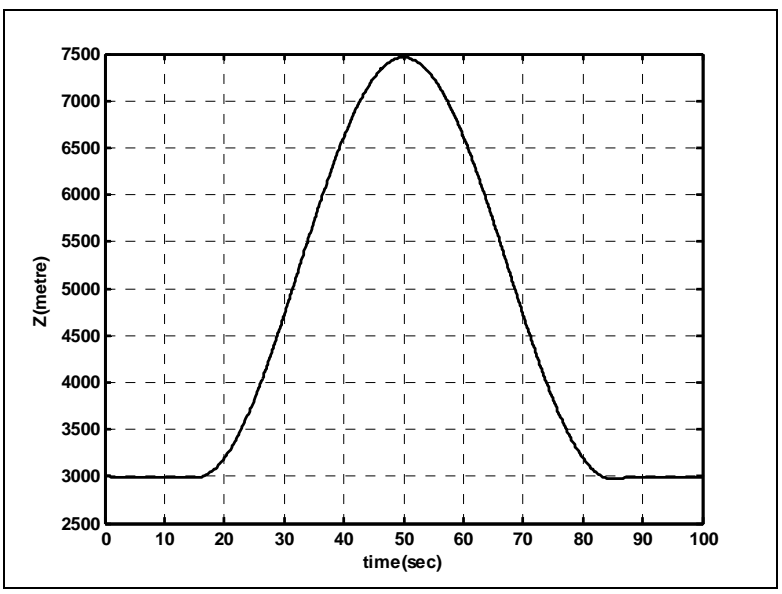

Fig.4. Vertical Distance

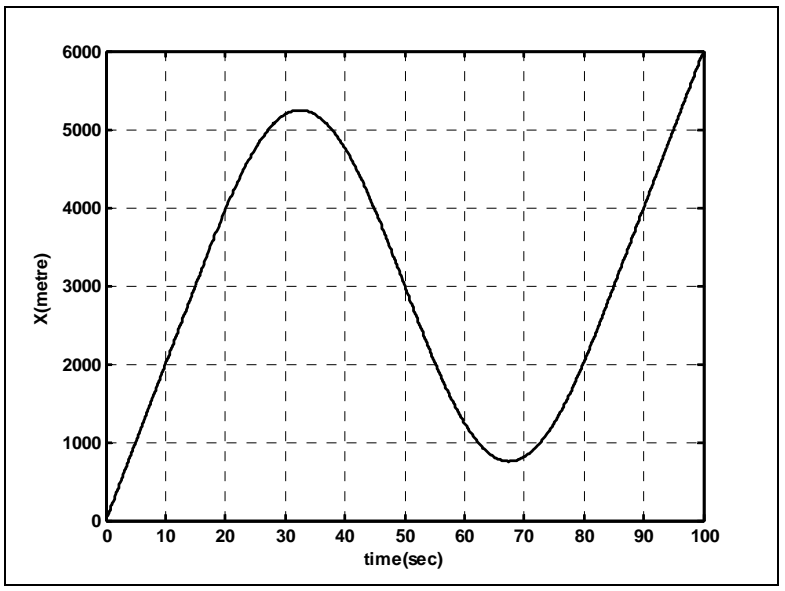

Fig.5. Horizontal Distance

Although the motion is in the longitudinal plane but we apply lateral disturbances and investigate the variation all over the motion. The disturbance shape with time is as shown in Fig.(6) and it has the amplitude and direction in the following sequence

No dist $=$ free of disturbance Fx dist $=1000 \mathrm{~N}$ in the $x$ direction Fy dist $=1000 \mathrm{~N}$ in the $y$ direction $\mathrm{Fz}$ dist $=1000 \mathrm{~N}$ in the $\mathrm{z}$ direction 


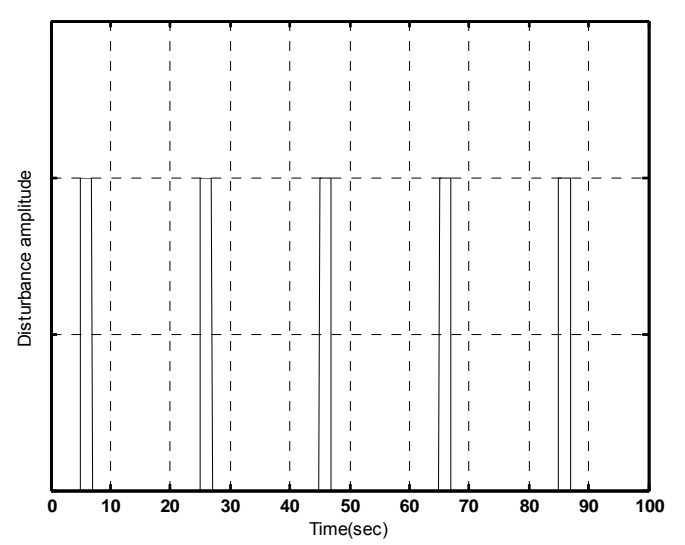

Fig.6. Disturbance Shape With Time

The following Figs show the motion parameters and the control laws during the maneuver with and without application of external disturbances.

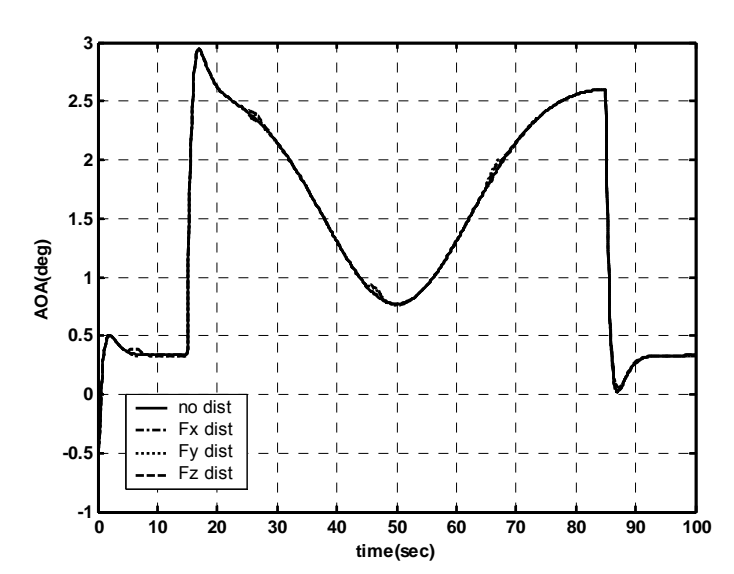

Fig.7. Angle Of Attack

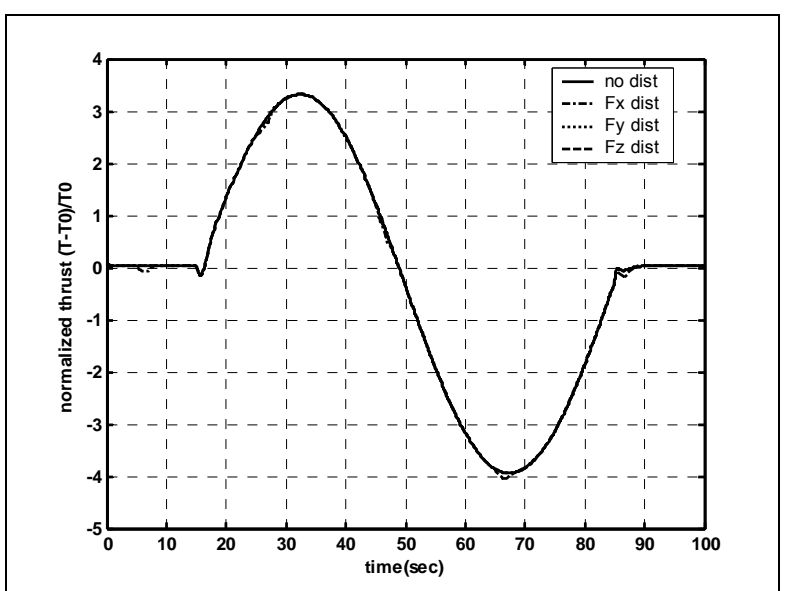

Fig.8. Normalized Thrust

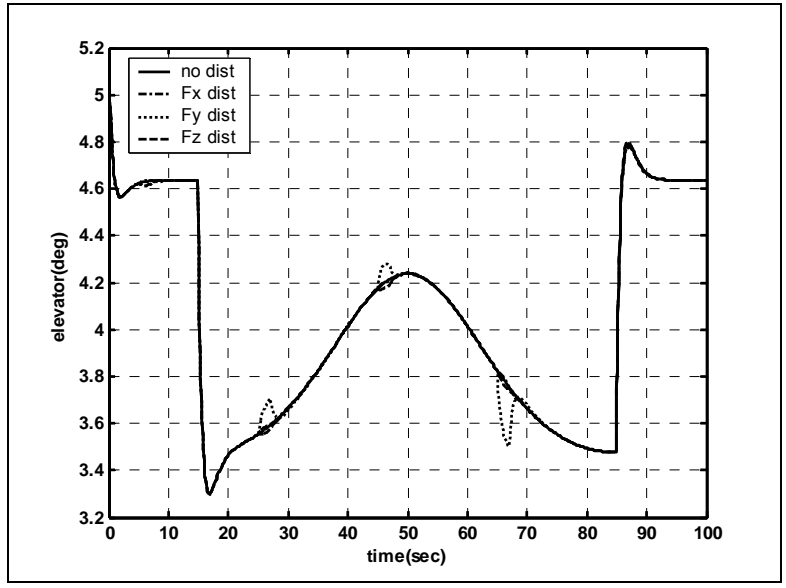

Fig.9. Elevator Deflection

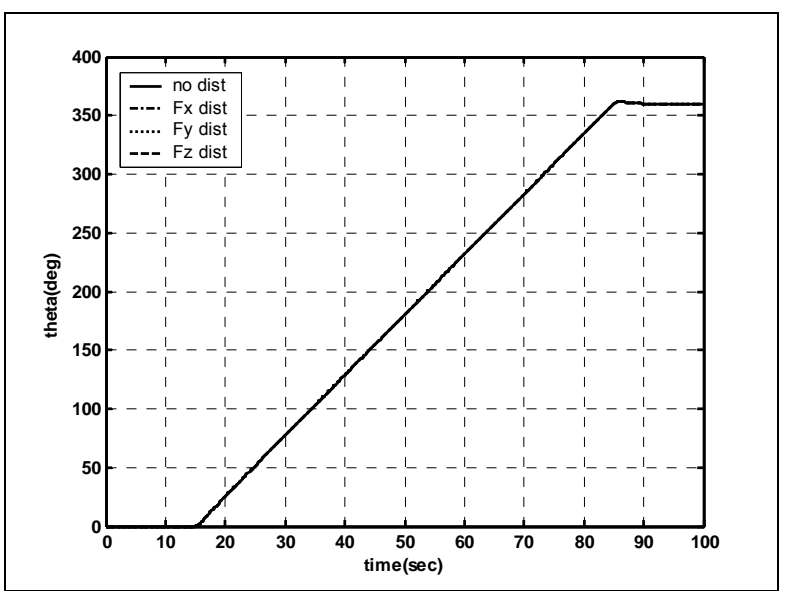

Fig.10. Pitch Angle

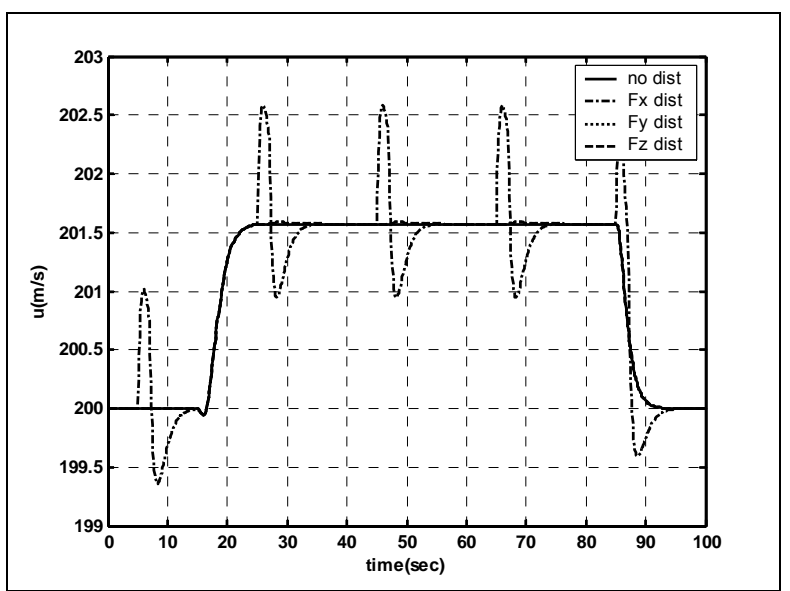

Fig.11. Velocity In X Body Axis 


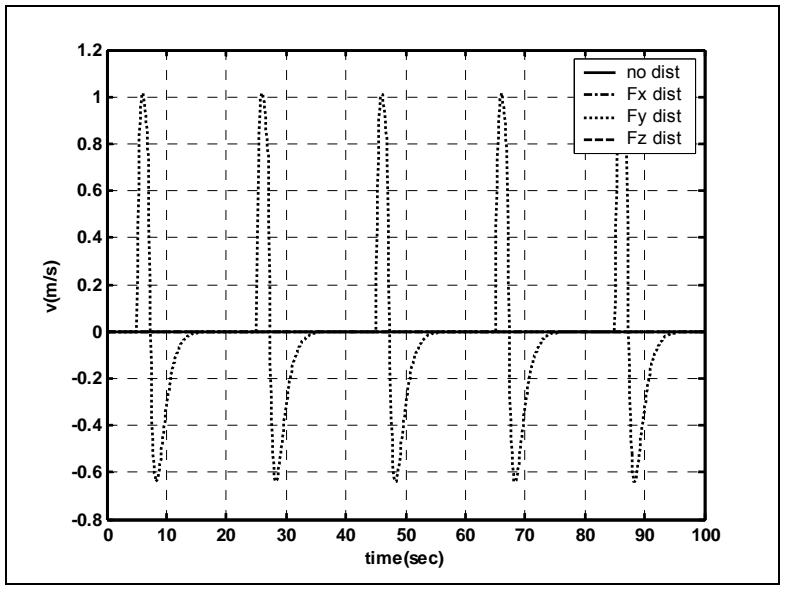

Fig.12. Velocity In Y Body Axis

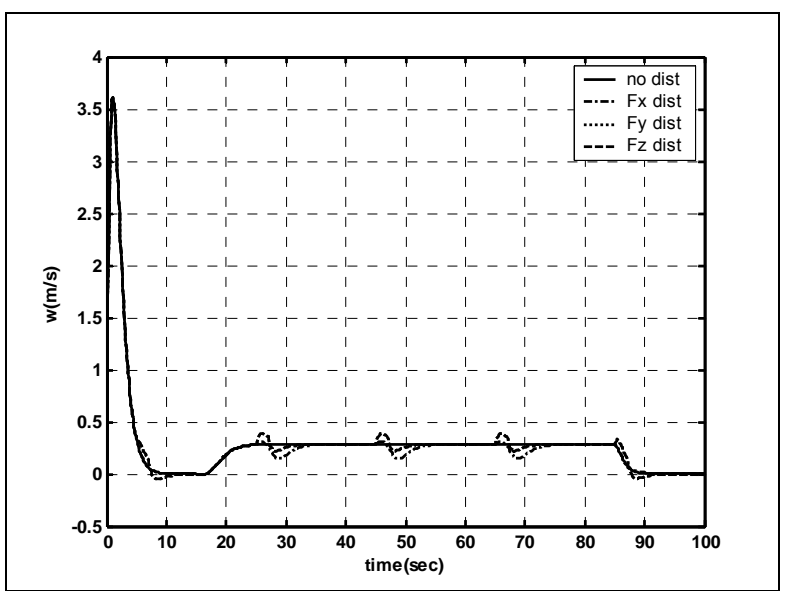

Fig.13. Velocity In Z Body Axis

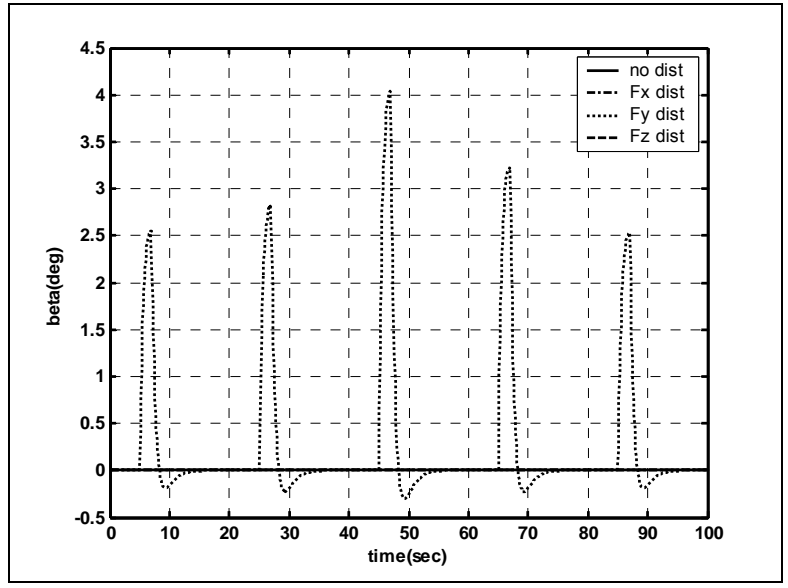

Fig.14. Side Slip Angle

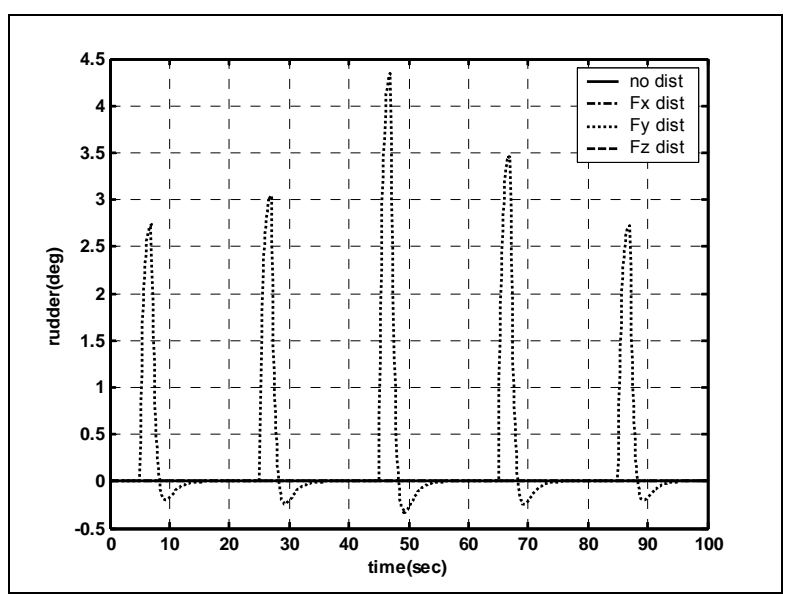

Fig.15. Rudder Deflection

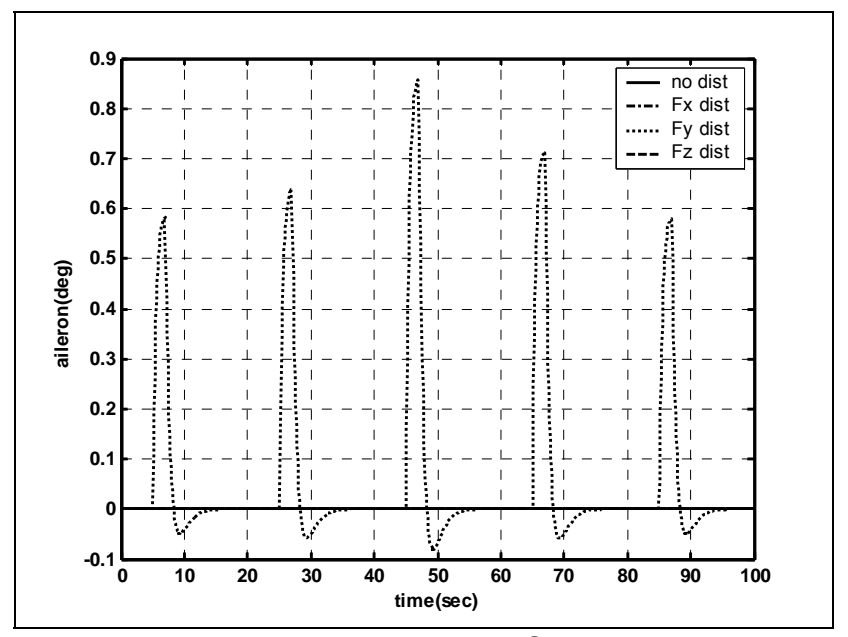

Fig.16. Aileron Deflection 
As shown in the above figures there is sudden change in motion parameters and control laws when the aircraft approaches the vertical loop and when gets out of it.

These sudden changes is due to the big difference between the aircraft state and the required trajectory parameters at that points which requires bigger values of forces and moments to overcome this differences and the algorithm interprets them into elevator deflections and thrust values which subsequently change the angle of attack. Although the motion is completely in the longitudinal plane, but when there is any lateral disturbance the algorithm activate the rudder and the aileron to overcome this disturbance as shown in Figs $(15,16)$

\section{Three Dimensional Application (Straight Line-Horizontal Loop-Straight Line with constant speed)}

The maneuver was performed with constant speed $V=200 \mathrm{~m} / \mathrm{s}$. the motion is combined longitudinal and lateral motion so the control is done by the four control elevator, rudder aileron and thrust and the lateral velocities and forces are taken into consideration.

the maneuver shape in $(x, y)$ plane and trajectory with time are as shown in Figs $(17,18,19)$

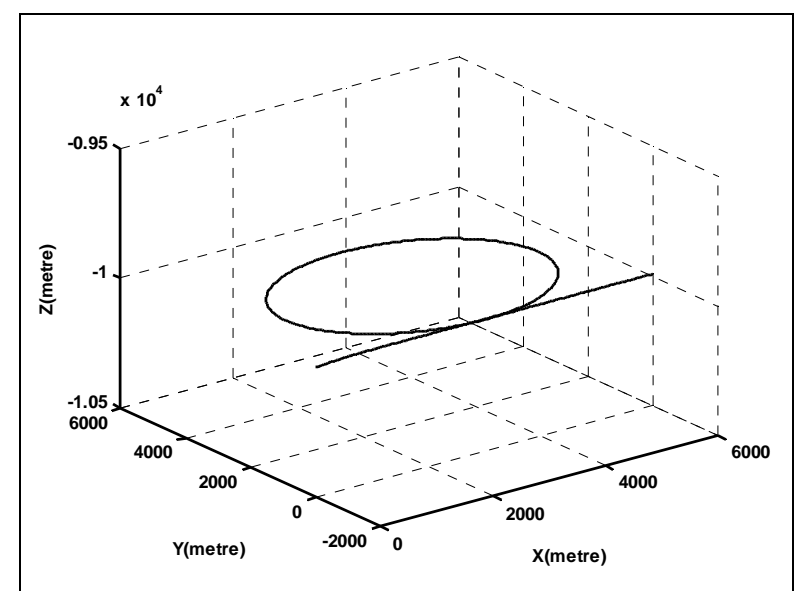

Fig.17. Straight Horizontal Loop Straight Maneuver

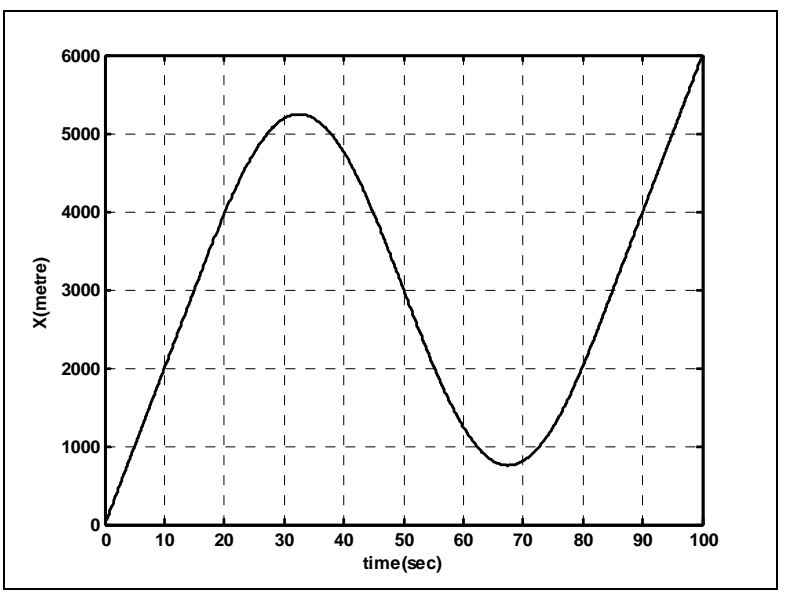

Fig.18. Distance In X Direction

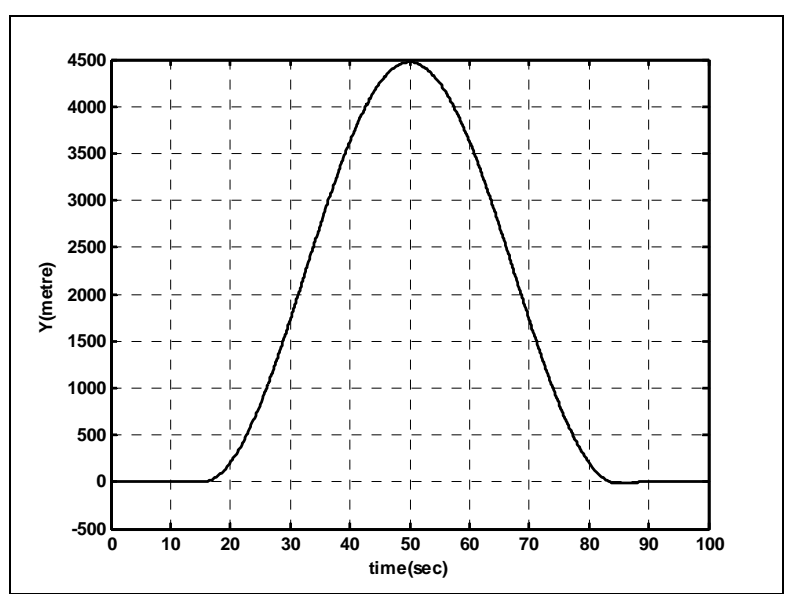

Fig.19. Distance In Y Direction

The disturbance shape with time is as shown in Fig.(20) and it has the

amplitude and direction in the following sequence

No dist $=$ free of disturbance

$\mathrm{Fx}, \mathrm{Mz}$ dist $=1000 \mathrm{~N}$.in $\mathrm{x}$ directions with 2000 N.m around $z$ axis

Fy,Mx dist $=1000 \mathrm{~N}$.in y directions with 2000 N.m around $x$ axis

Fz,My dist $=1000 \mathrm{~N}$.in z directions with 2000 N.m around y axis 


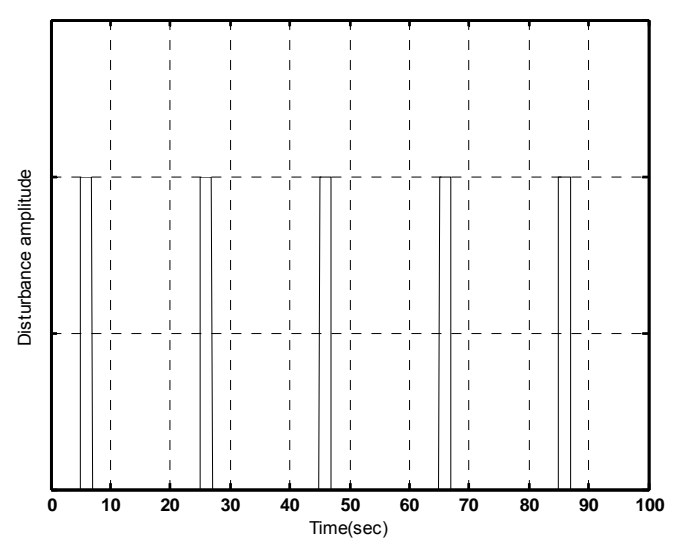

Fig.20. Disturbance Shape With Time

The following Figs show the motion parameters and the control laws during the maneuver with and without application of external disturbances.

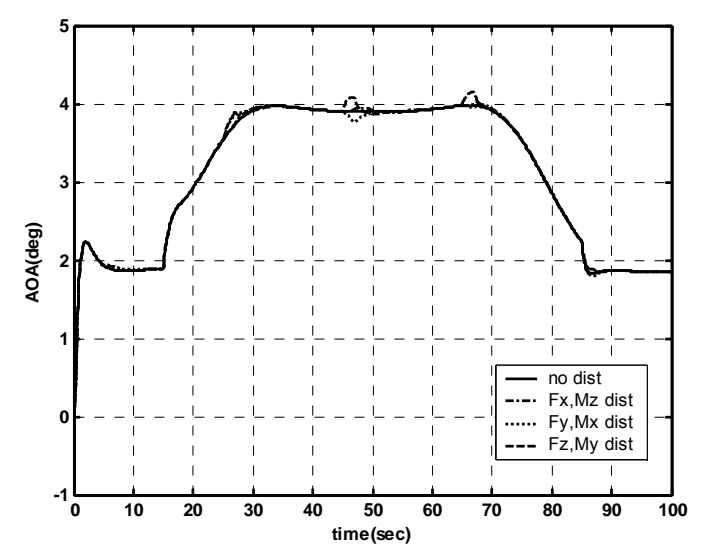

Fig.21. Angle Of Attack

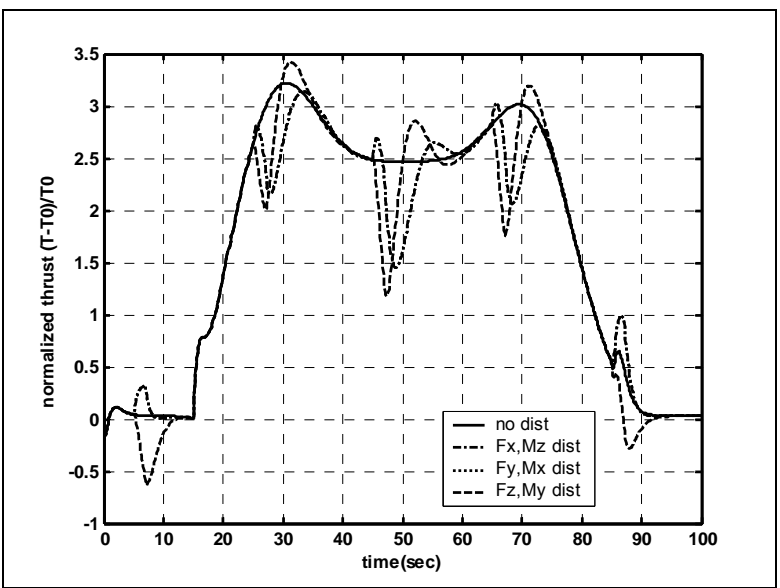

Fig.22. Normalized Thrust

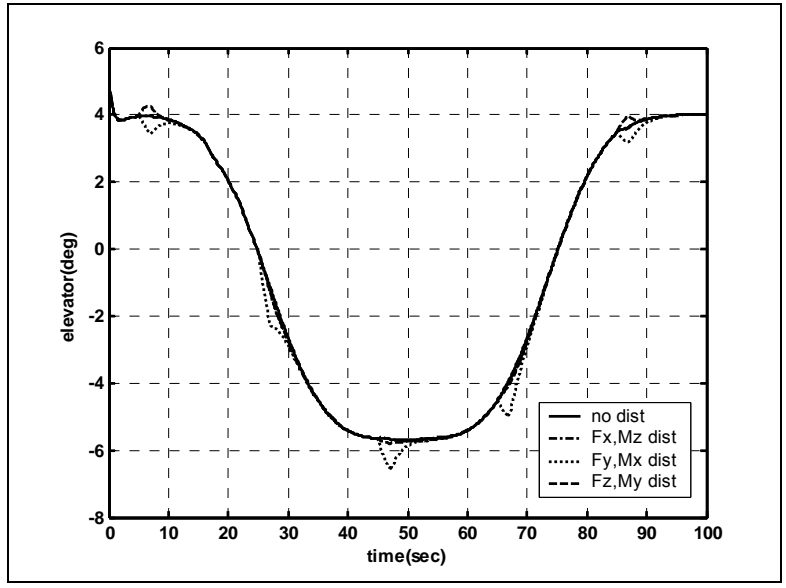

Fig.23. Elevator Deflection

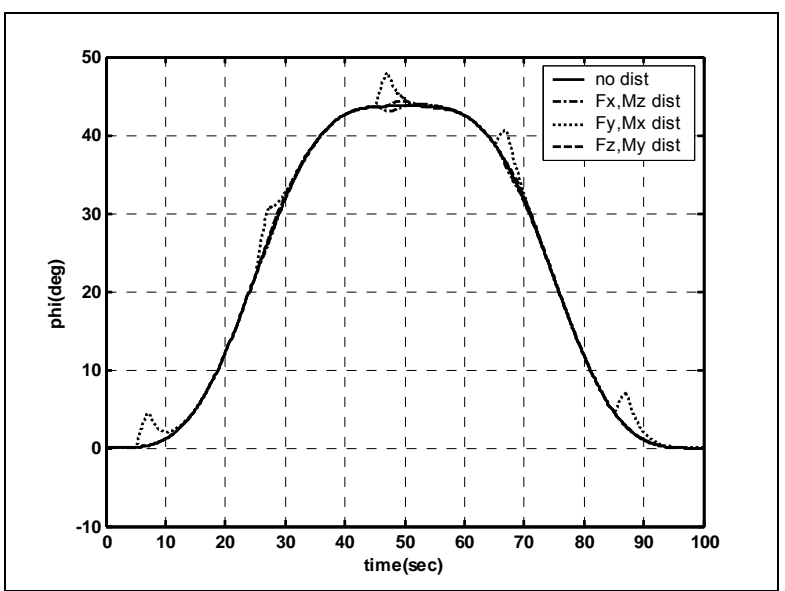

Fig.24. Roll Angle

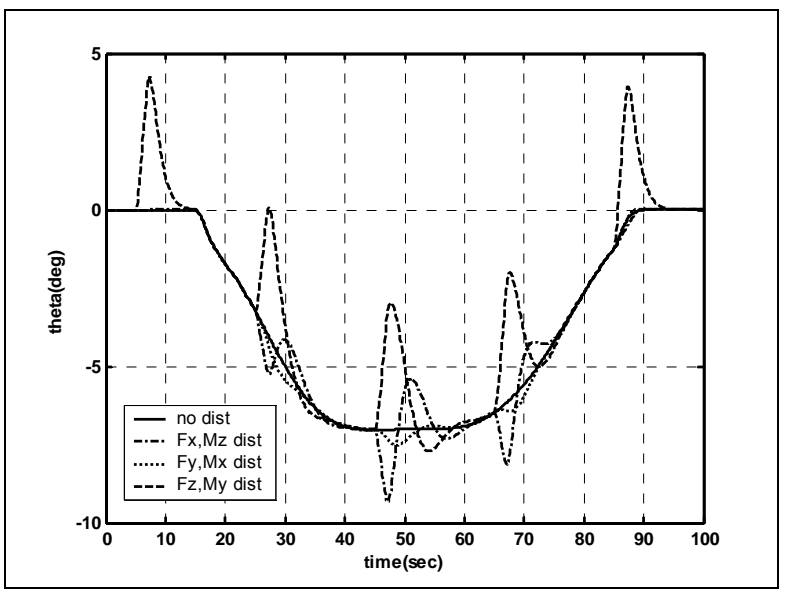

Fig.25. Pitch Angle 


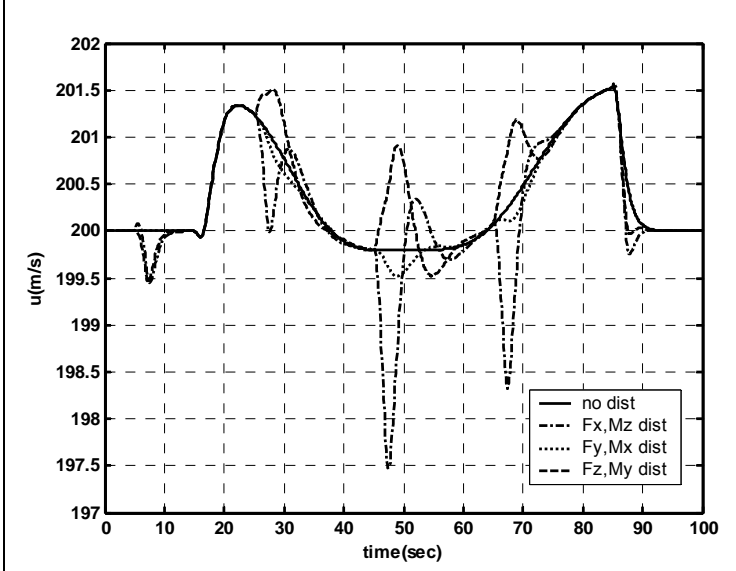

Fig.26. Velocity In X Body Axis

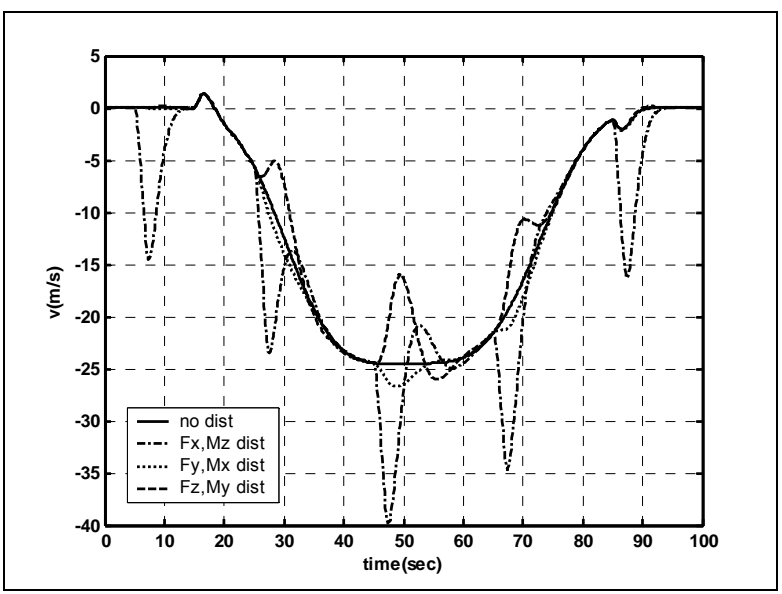

Fig.27. Velocity In Y Body Axis

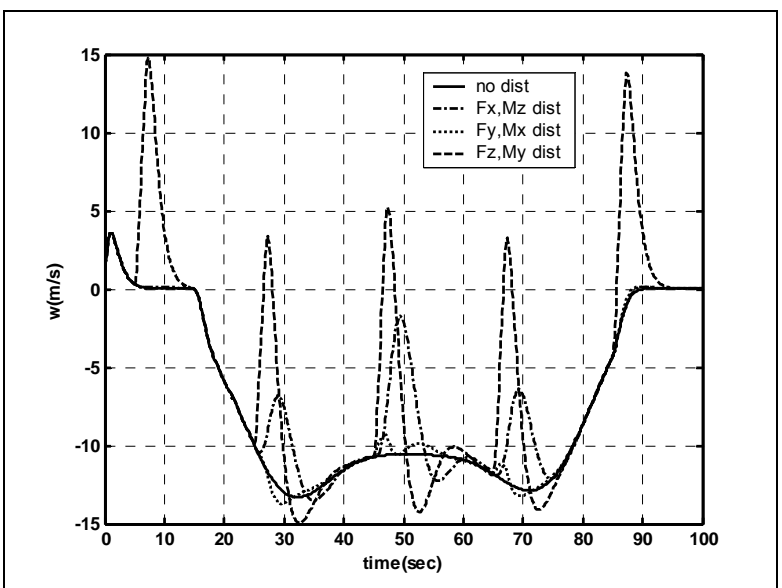

Fig.28. Velocity In Z Body Axis

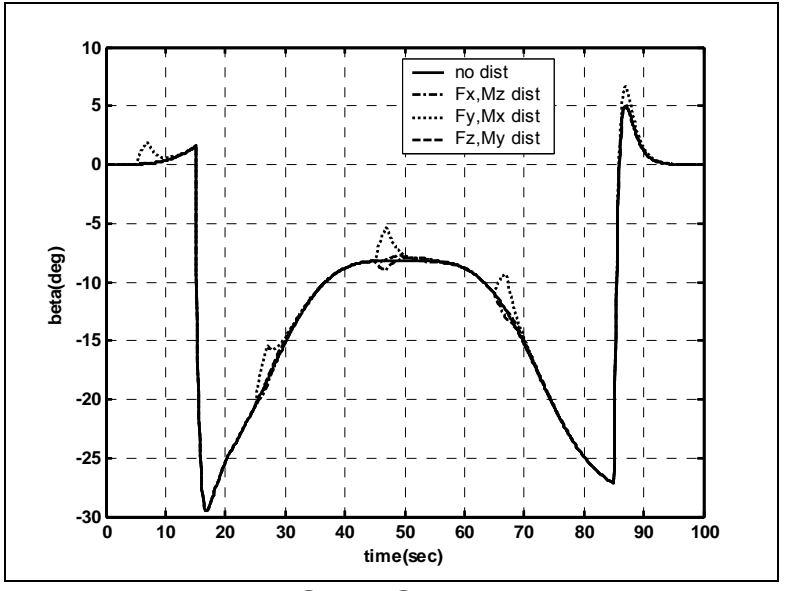

Fig.29. Side Slip Angle

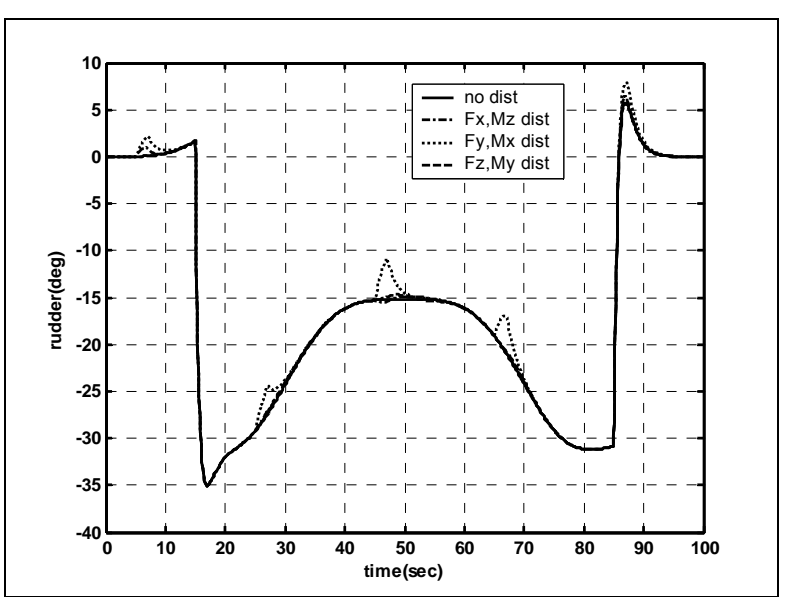

Fig.30. Rudder Deflection

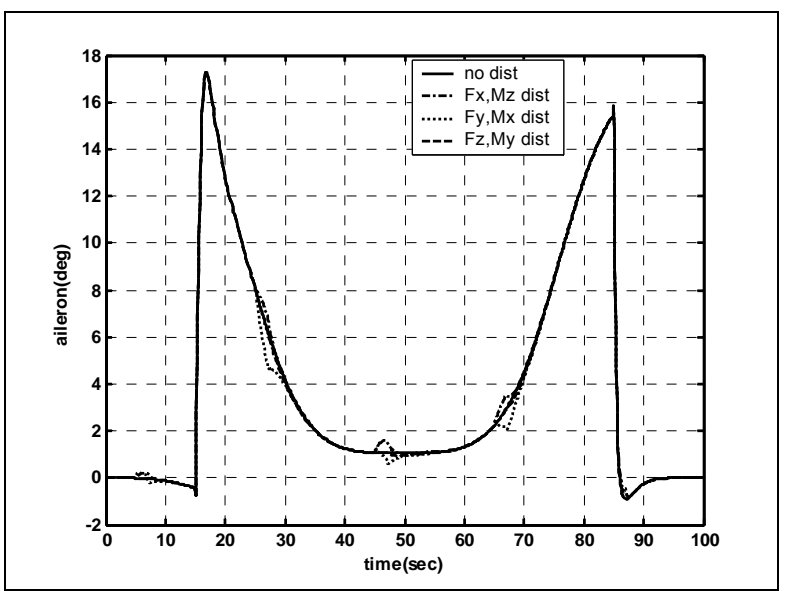

Fig.31. Aileron Deflection 
In the above figures it is clear that all the lateral motion parameters and controls are changing abruptly when approaching the horizontal loop especially the side slip angle and rudder deflection.

Note that the transient between the aircraft state and the required state can have better characteristics when the gain matrix of the error minimizing technique has another values [6] which can be designed based on different response characteristics or even have another control algorithm such as PID or optimal [8] control algorithm.

\section{CONCLUSION}

The proposed technique was successfully applied for two dimensional and three dimensional maneuvers . the following main conclusions can be drawn.

1- The technique gives valuable information on the nature of predetermined motion and the required control.

2- the technique has successfully shown the required action to overcome sudden disturbances

3- The technique doesn't need higher order derivatives of the predetermined trajectory unlike other inverse simulation techniques.

4- The technique is robust because in case of adding any external disturbance the algorithm performs the target maneuver and taking the required action to overcome this disturbance in consideration.

5- The technique is maneuver independent which means is applicable for any type of maneuvers no changes required in the algorithm for the change of the maneuver shape.

\section{REFERENCES}

[1] Irene M. Gregory "stability results of dynamic inversion devised to control large flexible aircraft " Paper No. 4284, AIAA guidance navigation and control conference, Montreal Canada, August 2001.

[2] Abdel Rahman M. M and Al-Bahi A. M "a generalized technique for inverse simulation of aircraft motion along predetermined trajectories ", Paper No. 3523, AIAA flight mechanics conference, Scottsdale, August 1994.

[3] James Robert Fisher "Aircraft control using nonlinear dynamic inversion in conjunction with adaptive robust control "master of science thesis, Texas A\&M University, Mechanical Engineering Department, December 2004.

[4] Mia Karlsson "control of unmanned aerial vehicle using nonlinear dynamic inversion " master of science thesis, Linkoping University, Electrical Engineering Department, June 2002. 
[5] John H. Blakelock ", Automatic Control Of Aircraft And Missiles", Wiley And Sons, 1991.

[6] Bandu N. Pamadi "Performance, Stability, Dynamics And Control Of Airplanes", AIAA education series, 1998.

[7] Nader Zaki Saweeris "aircraft inverse simulation along prescribed three dimensional trajectories" Master of science thesis, Cairo university, faculty of Engineering, Aerospace Department, September 1999.

[8] Ching-Fang Lin "Modern navigation, guidance and control processing", Printce Hall, 1991.

[9] Dong Chan Lee and Ramesh Agrawel "state feed back stabilization of an elastic aircraft motion", Paper No. 4254, AIAA guidance, Navigation and control conference, Denver ,August 2000. 